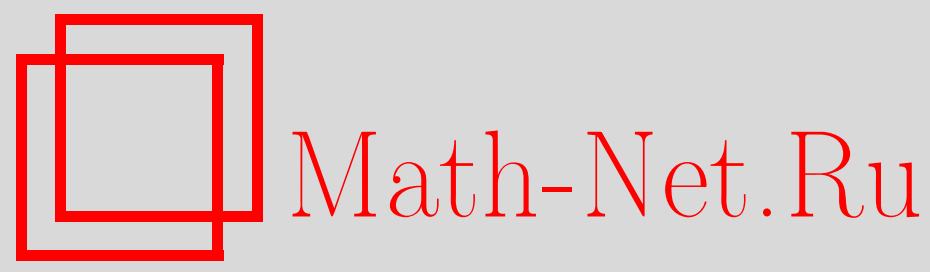

3. И. Бежаева, В. И. Оселедец, Вычисление энтропии для некоторой скрытой цепи Маркова, Дискрет. матем., 2012, том 24, выпуск 1, 108-122

DOI: https://doi.org/10.4213/dm1175

Использование Общероссийского математического портала Math-Net.Ru подразумевает, что вы прочитали и согласны с пользовательским соглашением http://www . mathnet.ru/rus/agreement

Параметры загрузки:

IP : 3.85 .73 .92

26 апреля 2023 г., $07: 44: 19$ 


\title{
Вычисление энтропии для некоторой скрытой цепи Маркова
}

\author{
() 2012 г. 3. И. Бежаева, В. И. Оселедец
}

В статье изучается один из вариантов задачи Эрдеша о распределении случайной величины

$$
\zeta=\sum_{i=1}^{\infty} \zeta_{i} \rho^{i},
$$

причем

$$
\mathbf{P}\left(\zeta_{i}=0\right)=q<1, \quad \mathbf{P}\left(\zeta_{i}=1\right)=p, \quad \rho \in[0,1], \quad \rho+\rho^{2}+\rho^{3}=1 .
$$

Определяются инвариантная мера Эрдеша на некотором компакте и соответствующая ей скрытая цепь Маркова, получена формула для вычисления энтропии инвариантной меры Эрдеша.

Работа выполнена при поддержке Российского фонда фундаментальных исследований, проект 07-01-92215-НЦНИЛ.

\section{1. Введение}

В [1] Эрдеш поставил задачу изучения функции распределения случайной величины

$$
\zeta=\sum_{i=1}^{\infty} \zeta_{i} \rho^{i} .
$$

Здесь $0<\rho<1$, и $\zeta_{1}, \zeta_{2}, \ldots$ - независимые одинаково распределенные величины, принимающие значения 0,1 , причем

$$
\mathbf{P}\left(\zeta_{i}=0\right)=\mathbf{P}\left(\zeta_{i}=1\right)=\frac{1}{2} .
$$

Задаче Эрдеша посвящено много статей. В [2] авторы дали определение меры Эрдеша на единичном интервале $[0,1]$, на компакте Фибоначчи, и инвариантной меры Эрдеша на компакте Фибоначчи для случая $\rho=(\sqrt{5}-1) / 2$.

В [2] было доказано, что мера Эрдеша эквивалентна инвариантной мере Эрдеша на компакте Фибоначчи. Кроме того, в этой же работе была поставлена и решена задача об эргодических свойствах инвариантной меры Эрдеша на компакте Фибоначчи.

В настоящей работе мы будем изучать распределение $\zeta$, когда

$$
\mathbf{P}\left(\zeta_{i}=0\right)=q<1, \quad \mathbf{P}\left(\zeta_{i}=1\right)=p,
$$


и $\rho \in[0,1]$ удовлетворяет уравнению

$$
\rho+\rho^{2}+\rho^{3}=1 .
$$

Меру, задаваемую $F(x)$ - функцией распределения случайной величины $\zeta-$ будем называть мерой Эрдеша на прямой.

По аналогии с компактом Фибоначчи, определим марковский компакт $X$ с алфавитом $\{0,1,2\}$ и набором допустимых слов $00,01,02,21$. Определим инвариантную меру Эрдеша на компакте $X$. Эта мера совпадает с распределением некоторой скрытой цепи Маркова. Будет получена формула для энтропии этой скрытой цепи Маркова. Таким образом, будет получена формула для вычисления энтропии инвариантной меры Эрдеша.

Отношение энтропии инвариантной меры Эрдеша и $\ln (\beta), \beta=1 / \rho$, есть хаусдорфова размерность инвариантной меры Эрдеша на компакте $X$ с метрикой

$$
d(x, y)=\rho^{n(x, y)},
$$

где $n(x, y)$ - длина наибольшего общего префикса слов $x$ и $y$. Эта размерность равна также хаусдорфовой размерности меры Эрдеша на прямой (см. [3, 4]).

Для нашего случая при $q=1 / 2$ полученная формула для хаусдорфовой размерности совпадает с формулой из [5] (см. также [6]).

Прямое вычисление энтропии по полученной нами формуле невозможно, так как ряды, входящие в формулу сходятся слишком медленно для эффективного вычисления. Поэтому в вычислениях использовалась процедура ускорения сходимости ряда. Для ускорения сходимости использовался метод, аналогичный методу из [7].

\section{2. Инвариантная мера Эрдеша на марковском компакте $X$}

Рассмотрим разбиение интервала $[0,1)$ на интервалы $\Delta_{0}=[0, \rho), \Delta_{1}=\left[\rho, \rho+\rho^{2}\right)$, $\Delta_{2}=\left[\rho+\rho^{2}, 1\right)$. Относительно отображения $V: x \mapsto\{\beta x\}$ это разбиение является марковским. Действительно, интервал $\Delta_{0}$ под действием отображения $V$ переходит в отрезок $[0,1)$, интервал $\Delta_{1}$ переходит в $\Delta_{0}$, а $\Delta_{2}$ в $\Delta_{1}$, то есть возможные переходы интервалов задаются матрицей инцидентности

$$
A=\left(\begin{array}{lll}
1 & 1 & 1 \\
1 & 0 & 0 \\
0 & 1 & 0
\end{array}\right) .
$$

Теперь каждой точке $x \in[0,1)$ сопоставим слово $x_{1} x_{2} \ldots$, где $x_{n}(x)=j$, если $V^{n-1} x \in \Delta_{j}, j=0,1,2$.

Пусть $X$ есть марковское множество бесконечных допустимых слов с алфавитом $\{0,1,2\}$. Мы называем слово допустимым, если все подслова длины 2 бесконечного допустимого слова задаются списком $\{00,01,02,10,21\}$. Множество $X$ образует компакт с метрикой $d(x, y)=\rho^{n(x, y)}$, где $n(x, y)$ есть длина наибольшего общего префикса слов $x$ и $y$.

Инвариантная мера Эрдеша на интервале $[0,1)$ (ее определение аналогично определению из [8]) переходит в инвариантную меру Эрдеша на $X$.

Аналогично [8] доказывается, что мера Эрдеша $d F(x)$ на прямой удовлетворяет уравнению

$$
d \tilde{F}(x)=M\left(x_{1}\right) d \tilde{F}(V x),
$$


где

$$
d \tilde{F}(x)=\left(d F(x), d F(x+\rho), d F\left(x+\rho+\rho^{2}\right), d F(x+1)\right)^{\top},
$$

а матрицы $M(0)=M_{0}, M(j)=M_{1}, j=1,2$, где

$$
M_{0}=\left(\begin{array}{cccc}
q & 0 & 0 & 0 \\
p & 0 & 0 & q \\
0 & p & 0 & 0 \\
0 & 0 & p & 0
\end{array}\right), \quad M_{1}=\left(\begin{array}{cccc}
p & 0 & 0 & q \\
p & 0 & 0 & p \\
0 & 0 & 0 & 0 \\
0 & 0 & 0 & 0
\end{array}\right)
$$

Введем квадратную матрицу 12-го порядка

$$
\begin{gathered}
M=\left(\begin{array}{lll}
M(00) & M(01) & M(02) \\
M(10) & M(11) & M(12) \\
M(20) & M(21) & M(22)
\end{array}\right), \\
M(00)=M(10)=M_{0}, \quad M(01)=M(02)=M(21)=M_{1} .
\end{gathered}
$$

Все остальные блоки матрицы $M$ нулевые. Спектральный радиус этой матрицы равен 1.

На пространстве бесконечных слов $x=x_{1} x_{2} \ldots \in X$ определим меру следующим образом:

$$
\begin{aligned}
\mu\left(\left\{x \in X: x_{1} \ldots x_{n}=a_{1} \ldots a_{n}\right)\right. & =L\left(a_{1}\right) M\left(a_{1}, a_{2}\right) \ldots M\left(a_{n-1}, a_{n}\right) R\left(a_{n}\right) /(L R), \quad n \geqslant 2, \\
\mu\left(x_{1}\right) & =L\left(x_{1}\right) R\left(x_{1}\right) /(L R),
\end{aligned}
$$

$L=(L(0), L(1), L(2))$ - левый собственный вектор, $R^{\top}=\left(R(0)^{\top}, R(1)^{\top}, R(2)^{\top}\right)-$ правый собственный вектор матрицы $M$ с собственным значением, равным 1.

Заметим, что мера $\mu$ инвариантна относительно сдвига $T$ в пространстве бесконечных допустимых слов с алфавитом $\{0,1,2\}$. Эту меру мы будем называть инвариантной мерой Эрдеша. Эта мера (см. [8]) совпадает с распределением скрытой марковской цепи, порождаемой матрицей $M$ и функцией склейки

$$
\begin{array}{ll}
g(i)=0, & i \in\{1,2,3,4\}, \\
g(i)=1, & i \in\{5,6,7,8\}, \\
g(i)=2, & i \in\{9,10,11,12\} .
\end{array}
$$

Вернемся к определенной выше мере $\mu$. Вектор $L$ имеет нулевые координаты с номерами $6,7,10,11$. У вектора $R$ две последние координаты нулевые. Поэтому инвариантная мера Эрдеша может быть получена при вычеркивании из матрицы $M$ и векторов $L, R$ столбцов и строк, соответствующих состояниям $6,7,10,11,12$. После вычеркивания из матрицы $M$ столбцов и строк с номерами 6,7 и 10, 11, 12 получим матрицу

$$
N=(n(i j), i, j=1, \ldots, 7)=\left(\begin{array}{ccccccc}
q & 0 & 0 & 0 & p & q & p \\
p & 0 & 0 & q & 0 & p & 0 \\
0 & p & 0 & 0 & 0 & 0 & 0 \\
0 & 0 & p & 0 & 0 & 0 & 0 \\
q & 0 & 0 & 0 & 0 & 0 & 0 \\
0 & 0 & p & 0 & 0 & 0 & 0 \\
0 & 0 & 0 & 0 & p & q & 0
\end{array}\right)
$$

Рассмотрим семь состояний, занумерованных числами $1,2,3,4,5,6,7$. Спектральный радиус матрицы $N$ равен 1 . Через $L=\left(L_{i}, i=1, \ldots, 7\right), R=\left(R_{i}, i=1, \ldots, 7\right)$ будем, 
как и раньше, обозначать, соответственно, правый и левый собственный вектор матрицы $N$ с собственным значением 1 .

Определим функцию $f$ от состояний $\{1,2,3,4,5,6,7\}$, полагая

$$
\begin{aligned}
& f(i)=0, \quad i=1,2,3,4 ; \\
& f(i)=1, \quad i=5,6 ; \\
& f(7)=2 .
\end{aligned}
$$

Эта функция и матрица $N$ определяют скрытую марковскую цепь (см. [8]).

Инвариантная мера Эрдеша $\mu-$ мера, порожденная этой скрытой марковской цепью на пространстве бесконечных допустимых слов $X$.

Матрицу $N$ представим в блочном виде

$$
N=\left(\begin{array}{lll}
N(00) & N(01) & N(02) \\
N(10) & N(11) & N(12) \\
N(20) & N(21) & N(22)
\end{array}\right),
$$

где

$$
\begin{array}{ll}
N(00)=\left(\begin{array}{llll}
q & 0 & 0 & 0 \\
p & 0 & 0 & q \\
0 & p & 0 & 0 \\
0 & 0 & p & 0
\end{array}\right), & N(01)=\left(\begin{array}{ll}
p & q \\
0 & p \\
0 & 0 \\
0 & 0
\end{array}\right), \\
N(02)=\left(\begin{array}{l}
p \\
0 \\
0 \\
0
\end{array}\right), & N(10)=\left(\begin{array}{llll}
q & 0 & 0 & 0 \\
0 & 0 & p & 0
\end{array}\right), \\
N(21)=\left(\begin{array}{ll}
p & q) .
\end{array}\right. &
\end{array}
$$

Все остальные матрицы $N(i j)$ нулевые.

Теперь мера $\mu$ можно задать с помощью блочной матрицы $N$ и векторов

$$
\begin{aligned}
L & =(L(0), L(1), L(2)), \\
L(0) & =\left(L_{1}, L_{2}, L_{3}, L_{4}\right)=\left(\left(1,(1-q)^{2},(1-q),(1-q)^{2} q\right),\right. \\
L(1) & =\left(L_{5}, L_{6}\right)=\left((2-q)(1-q), 1-q+2 q^{2}-q^{3}\right), \\
L(2) & =L_{7}=1-q, \\
R^{\top} & =\left(R(0)^{\top}, R(1)^{\top}, R(2)\right), \\
R(0) & =\left(R_{1}, R_{2}, R_{3}, R_{4}\right)^{\top}=\left((2-q) q,(1-q),(1-q)^{2},(1-q)^{3}\right)^{\top}, \\
R(1) & =\left(R_{5}, R_{6}\right)^{\top}=\left((2-q) q^{2},(1-q)^{3}\right)^{\top}, \\
R(2) & =R_{7}=q(1-q) .
\end{aligned}
$$

Наша основная цель состоит в вычислении энтропии $h$ инвариантной меры Эрдеша $\mu$.

\section{3. Вспомогательные обозначения и определения}

Рассмотрим граф с тремя вершинами 0, 1, 2 и ребрами 00, 01, 02, 10, 21. Спектральный радиус матрицы инцидентности этого графа равен $\beta$. 
Сопоставим ребру $i j$ матрицу $q p(i j)$, где $0<q<1, \alpha=p / q$,

$$
\begin{array}{ll}
p(00)=\left(\begin{array}{llll}
1 & 0 & 0 & 0 \\
\alpha & 0 & 0 & 1 \\
0 & \alpha & 0 & 0 \\
0 & 0 & \alpha & 0
\end{array}\right), & p(01)=\left(\begin{array}{ll}
\alpha & 1 \\
0 & \alpha \\
0 & 0 \\
0 & 0
\end{array}\right), \\
p(02)=\left(\begin{array}{l}
\alpha \\
0 \\
0 \\
0
\end{array}\right), & p(10)=\left(\begin{array}{llll}
1 & 0 & 0 & 0 \\
0 & 0 & \alpha & 0
\end{array}\right), \\
p(21)=\left(\begin{array}{ll}
\alpha & 1
\end{array}\right) . &
\end{array}
$$

Любой конечный путь графа есть конечное слово с буквами из алфавита $\{0,1,2\}$. Каждому пути в графе отвечает произведение матриц $q p(i j)$ вдоль этого пути. Для простых циклов с базовой вершиной 2 это произведение есть число, так как матрица $p(21)-$ это строка, а матрица $p(02)-$ столбец.

Каждое бесконечное слово, после первого попадания в 2, имеет вид $b_{1} 2 b_{2} \ldots$ Все слова $b_{j}$ устроены так: $1(0)^{k_{1}+1} \ldots 1(0)^{k_{s}+1}, k_{j} \geqslant 0$.

Рассмотрим простые циклы $1(0)^{k+1} 1$. Произведение матриц $q p(i j)$ вдоль такого цикла равно $q^{k+2} p(10) p(00)^{k} p(01)$. Назовем простой цикл распадным, если определитель матрицы этого цикла равен 0 и нераспадным в противном случае.

Для нераспадного цикла $k=3 n+1, n=0,1, \ldots$, и произведение матриц $q p(i j)$ вдоль такого цикла равно $q^{k+2} A(n)$, где

$$
A(n)=p(10) p(00)^{k} p(01)=\left(\begin{array}{cc}
\alpha & 1 \\
\alpha^{4}[n]_{\alpha^{2}} & \alpha^{3}[n+1]_{\alpha^{2}}
\end{array}\right) .
$$

В этом выражении

$$
[n]_{\alpha^{2}}=\frac{\alpha^{2 n}-1}{\alpha^{2}-1}
$$

Простейший распадный цикл получается при $k=0$. Ему отвечает матрица $q^{2}(1,0)^{\top}(\alpha, 1)$. При $k=3 n+2, k=3 n+3$ матрица распадного цикла равна

$$
q^{k+2}\left(1, \alpha^{3}[n+1]_{\alpha^{2}}\right)^{\top}(\alpha, 1)=q^{k+2} A(n)(0,1)^{\top}(\alpha, 1) .
$$

Введем обозначения

$$
\begin{aligned}
& p_{1}\left(n_{1} n_{2} \ldots n_{s}\right)=(\alpha, 1) A\left(n_{1}\right) \ldots A\left(n_{s}\right)(1,0)^{\top}, \\
& p_{2}\left(n_{1} n_{2} \ldots n_{s}\right)=(\alpha, 1) A\left(n_{1}\right) \ldots A\left(n_{s}\right)(0,1)^{\top} .
\end{aligned}
$$

Обозначим через $b^{\varepsilon}$ путь из 1 в 1 с ровно одним распадным циклом на конце:

$$
b^{\varepsilon}=1(0)^{k_{1}+1} 1(0)^{k_{2}+1} \ldots 1(0)^{k_{s-1}+1} 1(0)^{k_{s}+1},
$$

где $k_{j}=3 n_{j}+1, j=1, \ldots, s-1, k_{s} \neq 3 n_{s}+1, s \geqslant 1$.

Обозначим теперь через $b^{*}$ путь из 1 в 2 без распадных циклов:

$$
b^{*}=1(0)^{k_{1}+1} 1(0)^{k_{2}+1} \ldots 1(0)^{k_{s-1}+1} 1(0)^{k_{s}+1} 2 .
$$


Здесь также $k_{j}=3 n_{j}+1, j=1, \ldots, s-1$.

Все простые циклы из 2 можно представить в виде $b=b^{+} b^{*}$. При этом $b^{+}$либо пустое слово, либо $b^{+}=b_{1}^{\varepsilon} \ldots b_{t}^{\varepsilon}, t \geqslant 1$.

Определим $\alpha$-вес $p_{\alpha}\left(b^{\varepsilon}\right)$ пути $b^{\varepsilon}$ как

$$
p_{\alpha}\left(b^{\varepsilon}\right)=(\alpha, 1) A\left(n_{1}\right) \ldots A\left(n_{s}\right)(0,1)^{\top}=p_{2}\left(n_{1} \ldots n_{s}\right),
$$

если для распадного цикла $k_{s}=3 n_{s}+2, k=3 n_{s}+3, n_{s} \geqslant 0$.

Если для последнего распадного цикла $k_{s}=0$, то

$$
p_{\alpha}\left(b^{\varepsilon}\right)=(\alpha, 1) A\left(n_{1}\right) \ldots A\left(n_{s-1}\right)(1,0)^{\top}=p_{1}\left(n_{1} \ldots n_{s-1}\right), \quad s>1 .
$$

Если же $s=1, k_{1}=0$, то

$$
p_{\alpha}\left(b^{\varepsilon}\right)=(\alpha, 1)(1,0)^{\top}=\alpha .
$$

Для пути $b^{*}$ также определим $\alpha$-вес $p_{\alpha}\left(b^{*}\right)$. Тогда для $k_{s}=3 n_{s}+1, k_{s}=3 n_{s}$

$$
p_{\alpha}\left(b^{*}\right)=p_{1}\left(n_{1} \ldots n_{s}\right)=(\alpha, 1) A\left(n_{1}\right) \ldots A\left(n_{s}\right)(1,0)^{\top}, \quad n_{1} \geqslant 0, \ldots, n_{s} \geqslant 0 .
$$

При $k_{s}=3 n_{s}-1$

$$
p_{\alpha}\left(b^{*}\right)=p_{1}\left(n_{1} \ldots n_{s}\right)=(\alpha, 1) A\left(n_{1}\right) \ldots A\left(n_{s}\right)(1,0)^{\top}, \quad n_{1} \geqslant 0, \ldots, n_{s} \geqslant 1 .
$$

Пусть $\varphi(b)$ есть длина пути $b$, то есть число ребер пути $b$. Множитель $q^{\varphi(b)}$ назовем $q$-весом пути $b$. Этот вес мультипликативен относительно умножения путей.

Веса путей $b^{\varepsilon}, b^{*}$ определим как

$$
\begin{aligned}
& p\left(b^{\varepsilon}\right)=q^{\varphi\left(b^{\varepsilon}\right)} p_{\alpha}\left(b^{\varepsilon}\right), \\
& p\left(b^{*}\right)=q^{\varphi\left(b^{*}\right)} p_{\alpha}\left(b^{*}\right) .
\end{aligned}
$$

Вес пустого пути полагаем равным 1.

Из всех определений вытекает следующее утверждение.

Предложение 1. Условная мера Эрдеша простого иикла из 2 в 2 при условии, что траектории начинаются в 2, равна

$$
p(b)=q p\left(b^{+}\right) p\left(b^{*}\right) .
$$

Введем обозначения

$$
\begin{aligned}
& \Phi_{1}(z)=\sum_{s=1}^{\infty} \sum_{n_{1} \geqslant 0, \ldots, n_{s} \geqslant 0}(z q)^{3\left(n_{1}+\ldots+n_{s}+s\right)} p_{1}\left(n_{1} \ldots n_{s}\right), \\
& \Phi_{2}(z)=\sum_{s=1}^{\infty} \sum_{n_{1} \geqslant 0, \ldots, n_{s} \geqslant 0}(z q)^{3\left(n_{1}+\ldots+n_{s}+s\right)} p_{2}\left(n_{1} \ldots n_{s}\right) .
\end{aligned}
$$

Введем матрицу

$$
C=\left(\begin{array}{ll}
0 & 1 \\
1 & 0
\end{array}\right)
$$


Положим

$$
A_{0}(n)=C A(n) C \text {. }
$$

Тогда

$$
A_{0}(n)=\left(\begin{array}{cc}
\alpha^{3}[n+1]_{\alpha^{2}} & \alpha^{4}[n]_{\alpha^{2}} \\
1 & \alpha
\end{array}\right) .
$$

Теперь введем матрицы $M(0), M(1)$, полагая

$$
M(0)=\left(\begin{array}{cc}
\alpha^{2} & \alpha^{3} \\
0 & 1
\end{array}\right), \quad M(1)=\left(\begin{array}{cc}
\alpha^{3} & 0 \\
1 & \alpha
\end{array}\right) .
$$

Справедливо равенство

$$
A_{0}(n)=M(0)^{n} M(1) \text {. }
$$

Именно это представление приводит к представлению произведения $A_{0}\left(n_{1}\right) \ldots A_{0}\left(n_{s}\right)$ как произведения матриц $M(0), M(1)$ вдоль пути в бинарном дереве. Действительно,

$$
\begin{aligned}
p_{1}\left(n_{1} \ldots n_{s}\right) & =(\alpha, 1) C A_{0}\left(n_{1}\right) \ldots A_{0}\left(n_{s}\right) C(1,0)^{\top} \\
& =\alpha(1, \alpha) M(0)^{n_{1}} M(1) \ldots M(0)^{n_{s}}(0,1)^{\top} .
\end{aligned}
$$

Каждое слово $(0)^{n_{1}} 1(0)^{n_{2}} 1 \ldots(0)^{n_{s}}=d_{n-1}=\left(i_{1} \ldots i_{n-1}\right)$ есть двоичное слово длины $n_{1}+\ldots+n_{s}+s-1=n-1$. Поэтому можно считать, что $p_{1}\left(n_{1} \ldots n_{s}\right)=p_{1}\left(d_{n-1}\right)$. Если $n=1$, то $s=1, n_{1}=0$, и $p_{1}(0)=\alpha^{2}=\alpha(1, \alpha)(0,1)^{\top}$.

Пусть $M=M(0)+M(1), D_{n-1}$ есть множество двоичных слов длины $n-1$. Тогда

$$
\begin{aligned}
\Phi_{1}(z) & =\sum_{s=1}^{\infty} \sum_{n_{1} \geqslant 0, \ldots, n_{s} \geqslant 0}(z q)^{3\left(n_{1}+\ldots+n_{s}+s\right)} p_{1}\left(n_{1} \ldots n_{s}\right) \\
& =\sum_{n=1}^{\infty} \sum_{d \in D_{n-1}}(z q)^{3 n} p_{1}(d)=\alpha(1, \alpha) \sum_{n=1}^{\infty}(z q)^{3 n} M^{n-1}(0,1)^{\top} \\
& =\alpha(1, \alpha)(z q)^{3}\left(I d-(z q)^{3} M\right)^{-1}(0,1)^{\top}
\end{aligned}
$$

Аналогично,

$$
\begin{aligned}
p_{2}\left(n_{1} \ldots n_{s}\right) & =(\alpha, 1) C A_{0}\left(n_{1}\right) \ldots A_{0}\left(n_{s}\right) C(0,1)^{\top} \\
& =(1, \alpha) M(0)^{n_{1}} M(1) \ldots M(0)^{n_{s}}\left(\alpha^{3}, 1\right)^{\top} \\
& =p_{2}\left(n_{1} \ldots n_{s}\right)=p_{2}\left(d_{n-1}\right), \quad n>1 .
\end{aligned}
$$

При $n=1$

$$
p_{2}(0)=\alpha^{3}+\alpha=(1, \alpha)\left(\alpha^{3}, 1\right)^{\top} .
$$

Отсюда следует, что

$$
\Phi_{2}(z)=(1, \alpha)(z q)^{3}\left(I d-(z q)^{3} M\right)^{-1}\left(\alpha^{3}, 1\right)^{\top} .
$$




\section{4. Производящая функция $\varphi(b)$}

Производящая функция длины пути $\varphi(b), b=b^{+} b^{*}$, равна

$$
\begin{aligned}
\Phi(z)=\sum_{b} z^{\varphi(b)} p(b) & =q \sum_{b^{+}, b^{\varepsilon}}(z q)^{\varphi\left(b^{+} b^{*}\right)} p_{\alpha}\left(b^{+}\right) p_{\alpha}\left(b^{*}\right) \\
& =q \sum_{b^{+}}(z q)^{\varphi\left(b^{+}\right)} p_{\alpha}\left(b^{+}\right) \sum_{b^{*}}(z q)^{\varphi\left(b^{*}\right)} p_{\alpha}\left(b^{*}\right) .
\end{aligned}
$$

Нетрудно видеть, что

$$
\begin{aligned}
\Phi^{+}(z) & =\sum_{b^{+}}(z q)^{\varphi\left(b^{+}\right)} p_{\alpha}\left(b^{+}\right)=\sum_{b^{+}}(z q)^{\varphi\left(b^{+}\right)} p_{\alpha}\left(b^{+}\right) \\
& =1+\sum_{t=1}^{\infty} \sum_{b_{1}^{\varepsilon}} \ldots \sum_{b_{t}^{\varepsilon}}(z q)^{\varphi\left(b_{1}^{\varepsilon}\right)} p\left(b_{1}^{\varepsilon}\right) \ldots(z q)^{\varphi\left(b_{t}^{\varepsilon}\right)} p\left(b_{t}^{\varepsilon}\right) \\
& =1+\sum_{t=1}^{\infty}\left(\Phi^{\varepsilon}(z)\right)^{t}=\frac{1}{1-\Phi^{\varepsilon}(z)} .
\end{aligned}
$$

В этой формуле

$$
\Phi^{\varepsilon}(z)=\sum_{b_{t}^{\varepsilon}}(z q)^{\varphi\left(b^{\varepsilon}\right)} p\left(b^{\varepsilon}\right) .
$$

Для того, чтобы получить $\Phi^{+}(z)$, вычислим сначала $\Phi^{\varepsilon}(z)$. По определению,

$$
b^{\varepsilon}=1(0)^{k_{1}+1} 1(0)^{k_{2}+1} \ldots 1(0)^{k_{s-1}+1} 1(0)^{k_{s}},
$$

где $k_{j}=3 n_{j}+1, j=1, \ldots, s-1, k_{s} \neq 3 n_{s}+1, s \geqslant 1$.

При этом множество путей $b^{\varepsilon}$ можно разбить на три группы:

(1) $k_{s}=0$;

(2) $k_{s}=3 n_{s}+2$;

(3) $k_{s}=3 n_{s}+3, n_{s} \geqslant 0$.

Для двух последних случаев $\alpha$-вес $b^{\varepsilon}$ один и тот же и равен

$$
p_{\alpha}\left(b^{\varepsilon}\right)=p_{2}\left(n_{1} \ldots n_{s}\right), \quad n_{1} \geqslant 0, \ldots, n_{s} \geqslant 0 .
$$

Если $k_{s}=0$, то при $s>1 \alpha$-вес соответствующего пути равен

$$
p_{\alpha}\left(b^{\varepsilon}\right)=p_{1}\left(n_{1} \ldots n_{s-1}\right), \quad n_{1} \geqslant 0, \ldots, n_{s-1} \geqslant 0 .
$$

При $s=1, k_{s}=0$ мы получаем, что

$$
p_{\alpha}\left(b^{\varepsilon}\right)=\alpha .
$$

Поэтому

$$
\sum_{b^{\varepsilon}} z^{\varphi\left(b^{\varepsilon}\right)} p\left(b^{\varepsilon}\right)=S_{0}+S_{2}+S_{3}
$$


где $S_{0}, S_{2}, S_{3}$ соответствуют трем различным типам путей $b^{\varepsilon}$, где $k_{s}=0, k_{s}=3 n_{s}+2$, $k_{s}=3 n_{s}+3$ соответственно.

Нетрудно видеть, что

$$
\begin{aligned}
S_{0}(z) & =\alpha(z q)^{2}+\sum_{s=2}^{\infty} \sum_{n_{1} \geqslant 0, \ldots, n_{s-1} \geqslant 0}(z q)^{k_{1}+\ldots+k_{s-1}+2 s} p_{1}\left(n_{1} \ldots n_{s-1}\right) \\
& =\alpha(z q)^{2}+\sum_{s=1}^{\infty} \sum_{n_{1} \geqslant 0, \ldots, n_{s} \geqslant 0}(z q)^{3\left(n_{1}+\ldots+n_{s}+s\right)+2} p_{1}\left(n_{1} \ldots n_{s}\right) \\
& =\alpha(z q)^{2}+(z q)^{2} \Phi_{1}(z) .
\end{aligned}
$$

Далее,

$$
S_{2}(z)=\sum_{s=1}^{\infty} \sum_{n_{1} \geqslant 0, \ldots, n_{s} \geqslant 0}(z q)^{3\left(n_{1}+\ldots+n_{s}+s\right)+1} p_{2}\left(n_{1}, \ldots, n_{s}\right)=(z q) \Phi_{2}(z) .
$$

Наконец,

$$
S_{3}(z)=(z q)^{2} \Phi_{2}(z) .
$$

Проводя все вычисления, получаем, что

$$
\begin{aligned}
\Phi^{\varepsilon}(z) & =S_{0}(z)+S_{2}(z)+S_{3}(z) \\
& =\alpha(z q)^{2}+(z q)^{2} \Phi_{1}(z)+(z q)(1+(z q)) \Phi_{2}(z) .
\end{aligned}
$$

Теперь вычислим

$$
\Phi^{*}(z)=\sum_{b^{*}} z^{\varphi\left(b^{*}\right)} p\left(b^{*}\right)=V_{1}+V_{0}+V_{-1},
$$

также соответствующую трем типам путей $b^{*}$, где $k_{s}=3 n_{s}+1, k_{s}=3 n_{s}, n_{s} \geqslant 0$, и $k_{s}=3 n_{s}-1, n_{s} \geqslant 1$. Тогда

$$
V_{1}(z)=\sum_{s=1}^{\infty} \sum_{n_{1} \geqslant 0, \ldots, n_{s} \geqslant 0}(z q)^{k_{1}+k_{2}+\ldots+k_{s}+2 s} p_{1}\left(n_{1}, \ldots, n_{s}\right)=\Phi_{1}(z) .
$$

Далее,

$$
V_{0}(z)=(z q)^{-1} \Phi_{1}(z)
$$

Нетрудно видеть, что

$$
\begin{aligned}
V_{-1}(z)=(z q)^{-2} \sum_{s=1}^{\infty} \sum_{n_{1} \geqslant 0, \ldots, n_{s-1} \geqslant 0, n_{s} \geqslant 1}(z q)^{3\left(n_{1}+\ldots+n_{s}+s\right)} p_{1}\left(n_{1} \ldots n_{s}\right) \\
=(z q)^{-2}\left(\sum_{s=1}^{\infty} \sum_{n_{1} \geqslant 0, \ldots, n_{s} \geqslant 0}(z q)^{3\left(n_{1}+\ldots+n_{s}+s\right)} p_{1}\left(n_{1} \ldots n_{s}\right)\right. \\
\left.-\sum_{s=1}^{\infty} \sum_{n_{1} \geqslant 0, \ldots, n_{s-1} \geqslant 0}(z q)^{3\left(n_{1}+\ldots+n_{s}+s\right)} p_{1}\left(n_{1} \ldots 0\right)\right) .
\end{aligned}
$$

Можно показать, что

$$
p_{1}\left(n_{1}, \ldots, n_{s-1}, 0\right)=\alpha p_{1}\left(n_{1} \ldots n_{s-1}\right), \quad s \geqslant 2 .
$$


При $s=1$

$$
p_{1}(0)=\alpha^{2} \text {. }
$$

Поэтому

$$
V_{-1}(z)=\left[(z q)^{-2}-\alpha(z q)\right] \Phi_{1}(z)-\alpha^{2}(z q) .
$$

Отсюда получаем, что производящая функция для $\varphi\left(b^{*}\right)$ есть

$$
\Phi^{*}(z)=\left[1+(z q)^{-1}+(z q)^{-2}-\alpha(z q)\right] \Phi_{1}(z)-\alpha^{2}(z q) .
$$

Производящая функция $\Phi(z)$ равна

$$
\Phi(z)=q \frac{\Phi^{*}(z)}{1-\Phi^{\varepsilon}(z)}=\frac{q G_{1}(x)}{G_{2}(x)},
$$

где

$$
\begin{aligned}
G_{1}(x)= & (1-q)^{2} q z^{2}\left(1+(1-q)^{2} z^{2}-(1-q)^{2} z^{3}\right) \\
G_{2}(x)= & 1-z^{3}-q^{3}(2-3 z) z^{4}+q^{4}(1-z) z^{4} \\
& \quad+q^{2} z^{2}\left(1-z+z^{2}-3 z^{3}\right)-q\left(z+z^{2}-2 z^{3}-z^{5}\right) .
\end{aligned}
$$

Легко убедиться в том, что

$$
\Phi(1)=\sum_{b} p(b)=1
$$

Теперь нетрудно убедиться в том, что

$$
\mathbf{E} \varphi(b)=\Phi^{\prime}(1)=\frac{3-7 q+12 q^{2}-11 q^{3}+5 q^{4}-q^{5}}{(1-q)^{2} q} .
$$

Заметим, что $\mathbf{E} \varphi(b)+1$ есть среднее время первого возвращения из состояния 2 в 2 , и для инвариантной меры Эрдеша $\mu$ справедливо равенство

$$
\mu(2)=\frac{L(2) R(2)}{L R}=\frac{(1-q)^{2} q}{3-6 q+10 q^{2}-10 q^{3}+5 q^{4}-q^{5}}=\frac{1}{\mathbf{E} \varphi(b)+1} .
$$

Теперь вычисление энтропии инвариантной меры Эрдеша $h$ сводится к вычислению энтропии $H$ условного распределения на пространстве простых циклов с базовой вершиной 2, так как

$$
h=\mu(2) H=\frac{H}{\mathbf{E} \varphi(b)+1} \text {. }
$$

\section{5. Вычисление энтропии распределения на пространстве простых циклов с базовой вершиной 2}

Наша ближайшая цель состоит в получении удобной формулы для энтропии $H$ распределения на пространстве простых циклов с базовой вершиной 2. 
По определению,

$$
\begin{aligned}
H & =-\sum_{b} \log (q p(b)) q p(b) \\
& =-\log q \sum_{b}(1+\varphi(b)) q p(b)-q \sum_{b} \log \left(p_{\alpha}(b)\right) p(b) \\
& =-(1+E \varphi(b)) \log q-q \sum_{b}\left(\log \left(p_{\alpha}\left(b^{+}\right)\right)+\log \left(p_{\alpha}\left(b^{*}\right)\right)\right) p_{\alpha}\left(b^{+}\right) q^{\varphi\left(b^{+}\right)} p_{\alpha}\left(b^{*}\right) q^{\varphi\left(b^{*}\right)} .
\end{aligned}
$$

Вычислим отдельно последнюю сумму:

$$
\begin{aligned}
W & =\sum_{b}\left(\log \left(p_{\alpha}\left(b^{+}\right)\right)+\log \left(p_{\alpha}\left(b^{*}\right)\right)\right) p_{\alpha}\left(b^{+}\right) q^{\varphi\left(b^{+}\right)} p_{\alpha}\left(b^{*}\right) q^{\varphi\left(b^{*}\right)} \\
& =W^{+} P^{*}+W^{*} P^{+}
\end{aligned}
$$

где

$$
\begin{aligned}
W^{+} & =\sum_{b} \log \left(p_{\alpha}\left(b^{+}\right)\right) p_{\alpha}\left(b^{+}\right) q^{\varphi\left(b^{+}\right)}, & P^{+} & =\sum_{b^{+}} p\left(b^{+}\right) q^{\varphi\left(b^{+}\right)}=\Phi^{+}(1), \\
W^{*} & =\sum_{b} \log \left(p_{\alpha}\left(b^{*}\right)\right) p_{\alpha}\left(b^{*}\right) q^{\varphi\left(b^{*}\right)}, & P^{*} & =\sum_{b^{*}} p\left(b^{*}\right) q^{\varphi\left(b^{*}\right)}=\Phi^{*}(1) .
\end{aligned}
$$

При этом

$$
P^{+} P^{*}=\Phi^{+}(1) \Phi^{*}(1)=\frac{1}{q} \Phi(1)=\frac{1}{q} .
$$

Теперь

$$
W^{+}=\sum_{t=1}^{\infty} \sum_{j=1}^{t}\left(\sum_{b_{j}^{\varepsilon}} \log \left(p_{\alpha}\left(b_{j}^{\varepsilon}\right)\right) q^{\varphi\left(b_{j}^{\varepsilon}\right)} p_{\alpha}\left(b_{j}^{\varepsilon}\right)\right)\left(P^{\varepsilon}\right)^{t-1}=\frac{W^{\varepsilon}}{\left(1-P^{\varepsilon}\right)^{2}}
$$

где

$$
W^{\varepsilon}=\sum_{b^{\varepsilon}} \log \left(p_{\alpha}\left(b^{\varepsilon}\right)\right) p_{\alpha}\left(b^{\varepsilon}\right) q^{\varphi\left(b^{\varepsilon}\right)}, \quad P^{\varepsilon}=\sum_{b^{\varepsilon}} p_{\alpha}\left(b^{\varepsilon}\right) q^{\varphi\left(b^{\varepsilon}\right)} .
$$

Кроме того, было показано, что

$$
P^{+}=\frac{1}{1-P^{\varepsilon}}=\Phi^{+}(1) .
$$

Поэтому

$$
W^{+}=W^{\varepsilon}\left(P^{+}\right)^{2} \text {. }
$$

Отсюда

$$
W=P^{+}\left(W^{\varepsilon} P^{+} P^{*}+W^{*}\right)=P^{+}\left(\frac{1}{q} W^{\varepsilon}+W^{*}\right) .
$$

Мы воспользовались здесь тем, что $P^{+} P^{*}=1 / q$. Поэтому

$$
H=-(1+\mathbf{E} \varphi(b)) \log q-q W=-(1+\mathbf{E} \varphi(b)) \log q-P^{+}\left(W^{\varepsilon}+q W^{*}\right) .
$$


Положим

$$
\begin{aligned}
\sigma_{1} & =\sum_{s=1}^{\infty} \sum_{n_{1} \geqslant 0, \ldots, n_{s} \geqslant 0} \log \left(p_{1}\left(n_{1}, \ldots, n_{s}\right)\right) p_{1}\left(n_{1}, \ldots, n_{s}\right) q^{3\left(n_{1}+\ldots+n_{s}+s\right)}, \\
\sigma_{2} & =\sum_{s=1}^{\infty} \sum_{n_{1} \geqslant 0, \ldots, n_{s} \geqslant 0} \log \left(p_{2}\left(n_{1}, \ldots, n_{s}\right)\right) p_{2}\left(n_{1}, \ldots, n_{s}\right) q^{3\left(n_{1}+\ldots+n_{s}+s\right)} .
\end{aligned}
$$

По определению, $\alpha$-вес пути $b^{\varepsilon}$ равен

$$
\begin{array}{lll}
p_{\alpha}\left(b^{\varepsilon}\right)=p_{2}\left(n_{1}, \ldots, n_{s}\right), & k_{s}=3 n_{s}+2, & k_{s}=3 n_{s}+3, \quad n_{s} \geqslant 0, \\
p_{\alpha}\left(b^{\varepsilon}\right)=p_{1}\left(n_{1}, \ldots, n_{s-1}\right), & k_{s}=0, & s>1 .
\end{array}
$$

Если же $s=1, k_{s}=0$, то

$$
p_{\alpha}\left(b^{\varepsilon}\right)=\alpha .
$$

Поэтому, разбивая, как и раньше, выражение для $W^{\varepsilon}$ на три слагаемых, получаем, что

$$
W^{\varepsilon}=q^{2} \alpha \log (\alpha)+q^{2} \sigma_{1}+\left(q+q^{2}\right) \sigma_{2} .
$$

Теперь рассмотрим пути из 1 в 2 без распадных циклов. Такой путь имеет вид

$$
b^{*}=1(0)^{k_{1}+1} 1(0)^{k_{2}+1} \ldots 1(0)^{k_{s}+1} 2,
$$

и для таких путей $\alpha-$ вес, соответственно, равен

$$
\begin{array}{ll}
p_{\alpha}\left(b^{*}\right)=p_{1}\left(n_{1}, \ldots, n_{s}\right), & n_{1} \geqslant 0, \ldots, n_{s} \geqslant 0, \quad k_{s}=3 n_{s}+1, \quad k_{s}=3 n_{s}, \\
p_{\alpha}\left(b^{*}\right)=p_{1}\left(n_{1}, \ldots, n_{s}\right), & n_{1} \geqslant 0, \ldots, n_{s} \geqslant 1, \quad k_{s}=3 n_{s}-1 .
\end{array}
$$

Аналогично, формула для $W^{*}$ переписывается в виде

$$
\begin{aligned}
W^{*}= & \sum_{s=1}^{\infty} \sum_{n_{1} \geqslant 0, \ldots, n_{s} \geqslant 0} \log \left(p_{1}\left(n_{1}, \ldots, n_{s}\right)\right) p_{1}\left(n_{1}, \ldots, n_{s}\right) q^{3\left(n_{1}+\ldots+n_{s}+s\right)} \\
& +\sum_{s=1}^{\infty} \sum_{n_{1} \geqslant 0, \ldots, n_{s} \geqslant 0} \log \left(p_{1}\left(n_{1}, \ldots, n_{s}\right)\right) p_{1}\left(n_{1}, \ldots, n_{s}\right) q^{3\left(n_{1}+\ldots+n_{s}+s\right)-1} \\
& +\sum_{s=1}^{\infty} \sum_{n_{1} \geqslant 0, \ldots, n_{s} \geqslant 1} \log \left(p_{1}\left(n_{1}, \ldots . n_{s}\right)\right) p_{1}\left(n_{1}, \ldots, n_{s}\right) q^{3\left(n_{1}+\ldots+n_{s}+s\right)-2} .
\end{aligned}
$$

Справедливо равенство

$$
W^{*}=\sigma_{1}+\frac{1}{q} \sigma_{1}+\frac{1}{q^{2}}\left(\sigma_{1}-\sigma_{1}^{0}\right),
$$

где

$$
\sigma_{1}^{0}=\sum_{s=1}^{\infty} \sum_{n_{1} \geqslant 0, \ldots, n_{s-1} \geqslant 0} \log \left(p_{1}\left(n_{1}, \ldots, n_{s-1} 0\right)\right) p_{1}\left(n_{1}, \ldots, n_{s-1} 0\right) q^{3\left(n_{1}+\ldots+n_{s-1}+s\right)} .
$$

Напомним, что

$$
p_{1}\left(n_{1}, \ldots, n_{s-1}, 0\right)=\alpha p_{1}\left(n_{1}, \ldots, n_{s-1}\right), \quad s \geqslant 2 .
$$


При $s=1$

$$
p_{1}(0)=(\alpha, 1) A(0)(1,0)^{\top}=\alpha^{2} \text {. }
$$

Поэтому

$$
\sigma_{1}^{0}=2 \alpha^{2} q^{3} \log \alpha+q^{3} \alpha(\log \alpha) \Phi_{1}(1)+\alpha q^{3} \sigma_{1}
$$

Отсюда получаем, что

$$
H=c_{1} \sigma_{1}+c_{2} \sigma_{2}+c,
$$

где

$$
\begin{aligned}
c_{1} & =-\frac{2+3 q-q^{2}+4 q^{3}+3 q^{4}-5 q^{5}+4 q^{6}-2 q^{7}}{q-q^{4}}, \\
c_{2} & =-\frac{q\left(2+3 q-q^{2}+q^{4}-q^{5}\right)}{1-q^{3}}, \\
c & =\frac{a \log (1-q)-b \log q}{(1-q)^{2} q\left(1+q+q^{2}\right)} .
\end{aligned}
$$

При этом

$$
\begin{aligned}
& a=4 q-12 q^{2}+8 q^{3}+8 q^{4}-16 q^{5}+14 q^{6}-8 q^{7}+2 q^{8}, \\
& b=-3-q+5 q^{2}-2 q^{3}-13 q^{4}+22 q^{5}-18 q^{6}+9 q^{7}-2 q^{8} .
\end{aligned}
$$

При $q=1 / 2$ эти константы равны

$$
c=\frac{49}{4}, \quad c_{1}=-\frac{35}{4}, \quad c_{2}=-\frac{15}{8} .
$$

\section{6. Процедура ускорения сходимости рядов}

Итак, для вычисления энтропии необходимо вычислять ряды

$$
\begin{aligned}
\sigma_{1} & =\sum_{s=1}^{\infty} \sum_{n_{1} \geqslant 0, \ldots, n_{s} \geqslant 0} \log \left(p_{1}\left(n_{1}, \ldots, n_{s}\right)\right) p_{1}\left(n_{1}, \ldots, n_{s}\right) q^{3\left(n_{1}+\ldots+n_{s}+s\right)} \\
& =2 \alpha^{2} \log \alpha+q^{3} \sum_{n=2}^{\infty} \sum_{d_{n-1} \in D_{n-1}} \log \left(p_{1}\left(d_{n-1}\right)\right) p_{1}\left(d_{n-1}\right) q^{3 n} \\
& =\sum_{n=0}^{\infty} k_{n}^{(1)} q^{3(n+1)} \\
\sigma_{2} & =\sum_{s=1}^{\infty} \sum_{n_{1} \geqslant 0, \ldots, n_{s} \geqslant 0} \log \left(p_{2}\left(n_{1}, \ldots, n_{s}\right)\right) p_{2}\left(n_{1}, \ldots, n_{s}\right) q^{3\left(n_{1}+\ldots+n_{s}+s\right)} \\
& =\sum_{n=0}^{\infty} k_{n}^{(2)} q^{3(n+1)} .
\end{aligned}
$$


Таблица 1.

\begin{tabular}{cc}
\hline$q$ & $h_{d}$ \\
\hline 0,5 & $0,9804093195347986, n=15$ \\
& $0,9804093195347315, n=20$ \\
0,45 & $0,9771520623050443, n=15$ \\
& $0,9771520623047869, n=20$ \\
0,4 & $0,9664343579215019, n=15$ \\
& $0,9664343579213361, n=20$ \\
0,35 & $0,9456243417042867, n=15$ \\
& $0,9456243417042505, n=20$ \\
0,3 & $0,9108593564128254, n=15$ \\
& $0,9108593564128222, n=20$ \\
\hline
\end{tabular}

\begin{tabular}{cc}
\hline$q$ & $h_{d}$ \\
\hline 0,25 & $0,8574481002105665, n=15$ \\
& $0,8574481002105663, n=20$ \\
0,2 & $0,7800100418650452, n=15$ \\
& $0,7800100418650452, n=20$ \\
0,15 & $0,6721849172201769, n=15$ \\
& $0,6721849172201769, n=20$ \\
0,1 & $0,5254251828886658, n=15$ \\
& $0,5254251828886658, n=20$ \\
0,05 & $0,32442148594240267, n=15$ \\
& $0,32442148594240294, n=20$ \\
\hline
\end{tabular}

В этих формулах

$$
\begin{aligned}
& k_{n}^{(i)}=\sum_{d \in D_{n}} \log \left(p_{i}(d)\right) p_{i}(d), \quad i=1,2, \\
& k_{0}^{(1)}=2 \alpha^{2} \log \alpha, \\
& k_{0}^{(2)}=\left(\alpha^{3}+\alpha\right) \log \left(\alpha^{3}+\alpha\right) .
\end{aligned}
$$

Основная трудность при вычислении $H$ состоит в том, что ряды $\sum_{i=1}^{\infty} k_{n}^{(i)} q^{3 n}$ сходятся так медленно, что непригодны для численного счета. Следуя подходу Александера-Цагира (см. [7]), преобразуем этот ряд для ускорения сходимости.

Пусть имеется ряд $\sum_{n=1}^{\infty} k_{n} x^{n}$. Рассмотрим $s(\alpha)$ - максимальное по модулю собственное значение матрицы $M=M(0)+M(1)$. Тогда

$$
s(\alpha)=\frac{1}{2}\left(1+\alpha+\alpha^{2}+\alpha^{3}+\sqrt{1+2 \alpha-\alpha^{2}-\alpha^{4}+2 \alpha^{5}+\alpha^{6}}\right) .
$$

Введем величину

$$
\mu_{n}=k_{n}-s(\alpha) k_{n-1} .
$$

Тогда

$$
(1-s(\alpha) x)\left(\sum_{n=1}^{\infty} k_{n} x^{n}\right)=\sum_{n=1}^{\infty} \mu_{n} x^{n} .
$$

Рассмотрим

$$
\lambda_{n}=2 \lambda_{n-1}-\lambda_{n-2}+\mu_{n}-s(\alpha) \mu_{n-1} .
$$

Справедливы следующие соотношения между рядами:

$$
\begin{aligned}
\sum_{n=1}^{\infty} k_{n} x^{n} & =\frac{1}{1-s(\alpha) x} \sum_{n=1}^{\infty} \mu_{n} x^{n}, \\
(1-x)^{2} \sum_{n=1}^{\infty} \lambda_{n} x^{n} & =(1-s(\alpha) x) \sum_{n=1}^{\infty} \mu_{n} x^{n}, \\
\sum_{n=1}^{\infty} k_{n} x^{n} & =\frac{(1-x)^{2}}{(1-s(\alpha) x)^{2}} \sum_{n=1}^{\infty} \lambda_{n} x^{n} .
\end{aligned}
$$


Вычисление рядов

$$
k_{n}^{(i)}=\sum_{d \in D_{n}} \log \left(p_{i}(d)\right) p_{i}(d), \quad i=1,2,
$$

по этой схеме было сведено к вычислению рядов $\sum_{n=1}^{\infty} \lambda_{n} x^{n}, i=1,2$. Последние ряды сходятся намного быстрее.

В табл. 1 приведены значения

$$
h_{d}=\frac{h}{\log \beta}=\frac{H}{(\mathbf{E} \varphi(b)+1) \log \beta}
$$

хаусдорфовой размерности инвариантной меры Эрдеша.

\section{Список литературы}

1. Erdős P., On a family of symmetric Bernoulli convolutions. Amer. J. Math. (1939) 61, 974-975.

2. Sidorov N., Vershik A., Ergodic properties of the Erdös measures, the entropy of the goldenshift, and related problems. Monatsh. Math. (1998) 126, 215-261.

3. Lalley S. P., Random series in powers of algebraic integers: Hausdorff dimension of the limit distribution. J. London Math. Soc. (1998) 57, 628-654.

4. Бежаева 3. И., Оселедец В. И., Задача Эрдёша-Вершика для золотого сечения. Функииональный анализ и его приложения (2010) 44, №1, 3-13.

5. Grabner P. J., Kirschenhofer P., Tichy R. F., Combinatorial and arithmetical properties of linear numeration systems. Combinatorica (2002) 22, 245-267.

6. Feng De-Jun, The limited Rademacher function and Bernoulli convolutions associated with Pisot numbers. Adv. Math. (2005) 195, 24-101.

7. Alexander I. C., Zagier D., The entropy of a certain infinitely convolved Bernoulli measure. J. London Math. Soc. (1991) 44, 121-134.

8. Бежаева 3. И., Оселедец В. И., Меры Эрдёша, софические меры и марковские цепи. Записки научных семинаров ПОМИ (2005) 326, 28-47.

Статья поступила 21.01.2011. 